1997 Particle Accelerator Conf., Vancouver, B.C., Canada, May 12-16, 1997.

$$
\text { CONF-970503--126 }
$$

\title{
Overcoming Weak Intrinsic Depolarizing Resonances with Energy-jump*
}

\author{
H. Huang ${ }^{1}$, L. Ahrens ${ }^{1}$, J.G. Alessi ${ }^{1}$, M. Bai ${ }^{2.3}$, K.A. Brown ${ }^{1}$, G. Bunce ${ }^{1}$, \\ P. Cameron ${ }^{1}$, P. Ingrassia' ${ }^{1}$ A.E. Kponou ${ }^{1}$, K. Krueger ${ }^{3}$, S.Y. Lee ${ }^{2}$, \\ A.U. Luccio ${ }^{1}$, Y.I. Makdisi' , F. Mariam ${ }^{1}$, M. Okamura ${ }^{5}$, L. Ratner ${ }^{1}$, \\ K. Reece ${ }^{1}$, T. Roser ${ }^{1}$, H. Sato ${ }^{4}$, H.M. Spinka ${ }^{3}$, N. Tsoupas ${ }^{1}$, \\ D.G. Underwood ${ }^{3}$, W. van Asselt ${ }^{1}$, N.W. Williams ${ }^{1}$, and A. Yokosawa ${ }^{3}$ \\ (1) Brookhaven National Laboratory, Upton, NY 11973, USA \\ (2) Department of Physics, Indiana University, Bloomington, IN 47405, USA \\ (3) Argonne National Laboratory, 9700 Cass Ave., Argonne, IL 60493, USA \\ RECEIVED
}

(4) KEK, I-1 Oho, Tsukuba-shi, Ibaraki-ken, 305, Japan

(5) RIKEN, Wako, Saitama, 351-01, Japan

\begin{abstract}
In the recent polarized proton runs in the AGS, a 5\% partial snake was used successfully to overcome the imperfection depolarizing resonances. Polarized proton beam was accelerated up to the required RHIC injection energy of $25 \mathrm{GeV}$. However, significant amount of polarization was lost at $0+\nu_{y}, 12+\nu_{y}$ and $36+\nu_{y}$, which is believed to be partially due to the coupling resonances. To overcome the coupling resonance, an energy-jump was generated by rapidly changing the beam circumference using the powerful AGS if system. It clearly demonstrates that the novel energy-jump method can successfully overcome coupling resonances and weak intrinsic resonances.
\end{abstract}

\section{INTRODUCTION}

The spin vector of an orbiting particle in a synchrotron obeys the Thomas-BMT equation [1], where the dynamics of spin motion can be characterized by the spin tune $\nu_{s}$, which is defined as the number of spin precessions per revolution about a stable spin direction. The acceleration of polarized beam will encounter depolarizing resonances whenever the spin precession frequency exactly matches the frequency with which the protons encounter depolarizing horizontal magnetic fields. For an ideal accelerator, where orbiting particles experience only the vertical guide field, the spin tune is equal to $G \%$, where $G=1.7928$ is the anomalous $G$-factor of the proton, and $\gamma$ is the relativistic Lorentz factor. The resonance condition for imperfection depolarizing resonances arises when $\nu_{s}=G_{\gamma}=n$, where $n$ is an integer. The condition for intrinsic depolarizing resonances arises when $\nu_{s}=G_{\gamma}=k P \pm \nu_{y}$, where $k$ is an integer, $\nu_{y}$ is the vertical betatron tune, and $P$ is the superperiodicity. For the Brookhaven Alternating Gradient Synchrotron (AGS), $P=12$ and $\nu_{y} \approx 8.8$.

Traditionally, the imperfection depolarizing resonances are compensated with the tedious harmonic correction

\footnotetext{
- Work performed under the auspices of the U.S. Dept. of Energy
}

method and the intrinsic depolarizing resonances are overcomed with aune-jump method[2]. The polarized proton runs of experiment E-880 at the AGS aim to demonstrate the feasibility of polarized proton acceleration using a 5\% partial Siberian snake [3]. In the first run it was shown that a $5 \%$ snake is sufficient to avoid depolarization due to the imperfection resonances [4]. The results are shown in Fig. 1 as open circles and dashed lines. The pulsed tunejump quadrupoles were not powered in this run. Although some depolarization at intrinsic resonances are expected, the level of the depolarization does not agree with a simple model calculation. A spin tracking study using SPINK [5] was then performed and it showed that there is an extra resonance adjacent to the intrinsic resonance which causes further depolarization [6]. This additional resonance can be easily understood as a linear coupling effect. Since the solenoidal $5 \%$ partial snake introduces considerable linear coupling between the two transverse betatron motions, the vertical betatron motion has also a component with the horizontal betatron frequency. As a consequence, the beam will see an additional resonance, the so-cailed coupling resonance at $G_{\gamma}=0+\nu_{x}$, besides the intrinsic resonance at $G \gamma=0+\nu_{y}$. In addition to the linear coupling effect, the synchrotron motion also causes some additional depolarization but to a smaller extent. The second run showed that it is possible to use the tune jump method in the presence of the partial snake. A new record high energy for accelerated polarized proton beam of $25 \mathrm{GeV}$ was reached with about $12 \%$ beam polarization left. Again no polarization was lost due to the imperfection resonances and depolarization from most intrinsic resonances was avoided with the tune jump quadrupoles. However, as can be seen from Fig. 1, significant amount of polarization was lost at $G \gamma=0+\nu_{y}, 12+\nu_{y}$ and $36+\nu_{y}$. The first two of these three resonances were successfully crossed previously when the partial snake was not installed. It is believed that the losses are partially due to the coupling resonances. The tune jump method changes the vertical betatron tune within less than one revolution

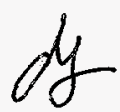




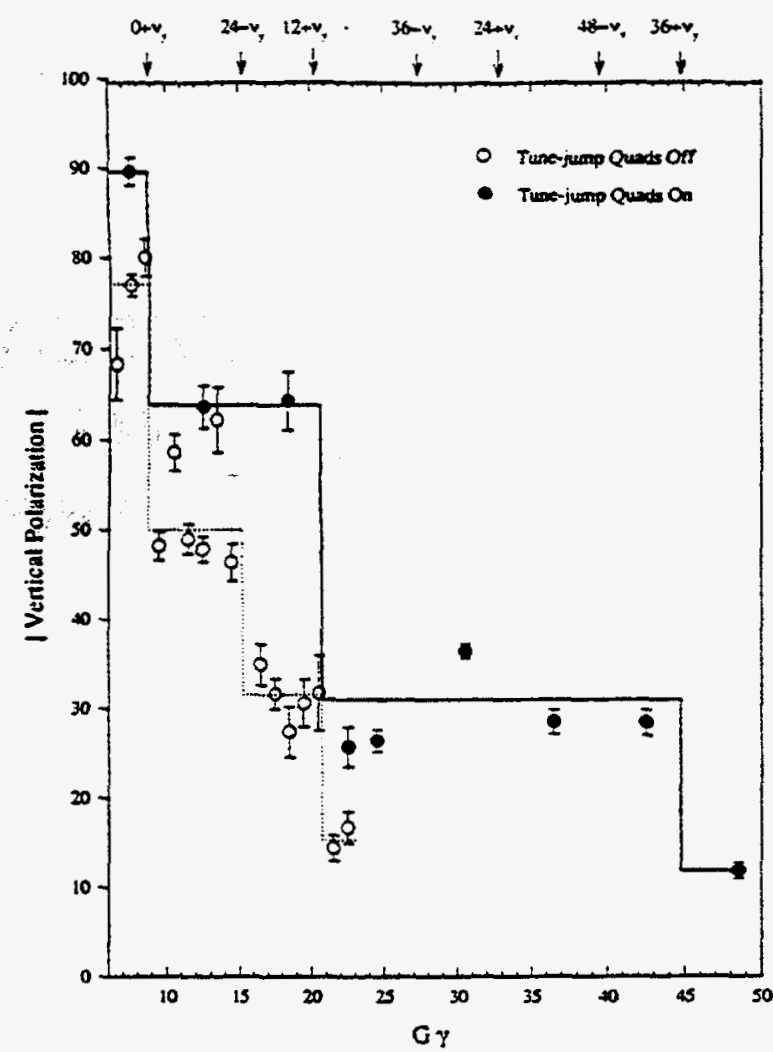

Figure 1: Results of AGS $5 \%$ partial snake test. The measured absolute value of the vertical polarization at $G \gamma=$ $n+\frac{1}{2}$ up to $G \gamma=48.5$. The open circles are the results without the tune-jump quadrupoles and the solid points are the results with the tune-jump quadrupoles.

to effectively make the resonance crossing speed very fast. However, the coupling resonance is still crossed at the normal crossing speed and can cause polarization loss. The strength of the tune jump quadrupoles is not sufficient to jump the last resonance $G \gamma=36+\nu_{y}$. Instead, we attempted to induce spin flip at this resonance but were only partially successful. A spin tracking shows that the amount of depolarization observed agrees with the effect of the strong coupling resonance at $G \gamma=36+\nu_{x}$.

\section{OVERCOMING THE COUPLING RESONANCES}

As shown in the simulations, it is clear that the unexpected depolarization level after crossing the strong intrinsic resonances is due to the combined effect of the coupling resonance and synchrotron oscillation. The coupling resonance strength can be decreased by separating the tune. However, separating the two tunes for more than 0.5 unit does not help, because the source of the coupling is localized at the snake solenoid. The ratio of the coupling resonance strength to the intrinsic resonance strength with a 5\% partial snake and 0.1 unit tune separation is about 0.06 . With a larger rune separation of 0.3 , the ratio goes down to 0.03 . Also, since the coupling resonances are generally weak, the acceleration rate can be maximized to minimize the depolarization at the coupling resonance. With a $25 \pi$ $\mathrm{mm}$-mrad polarized beam in the AGS, the coupling resonances adjacent to the weak intrinsic resonances such as $24-\nu_{y}, 24+\nu_{y}$ and $48-\nu_{y}$ will have little effect with the regular acceleration rate $\alpha=4.5 \times 10^{-5}$. But the coupling resonances adjacent to the strong intrinsic resonances such as $0+\nu_{y}, 12+\nu_{y}, 36-\nu_{y}$ and $36+\nu_{y}$ will cause significant depolarization. This ratio makes it impossible to cross them at a single speed without losing polarization. Using a very slow crossing speed could fully flip spin after the intrinsic resonance, but on the other hand, it also increases the depolarization from the coupling resonances. Even the traditional tune-jump quadrupole method is challenged. The tune jump method changes the betatron tunes in less than one orbit turn to effectively make the resonance crossing speed very fast. If the two tunes are separated, the coupling resonance is crossed by the beam at the normal crossing speed which causes depolarization. Moreover, the tune jump can increase the beam emirtance and increase the strengths of subsequent coupling and inurinsic resonances. In short, the benefits of all these methods will be reduced or diminished without extra efforts to overcome the coupling resonances.

To eliminate the coupling resonances, one can think of compensating the linear coupling globally. Two sets of strong skew quadrupoles could compensate the linear coupling of the solenoidal partial snake globally. However, with a global decoupling, the orbit is flat globally but not locally, which means that at certain locations, the coupling will be non-zero and may still cause some problem. One possible way is to eliminate only the coupling resonance closest to the intrinsic resonance instead of eliminating everyone. Some further simulation is needed to test the idea Practically, the skew quadrupoles in the AGS are not strong enough to compensate the coupling of the solenoidal snake.

When the intrinsic resonances are crossed with coherent betatron oscillation excited with an AC dipole [7], slow acceleration rare or tune-jump, the coupling resonances can be crossed using a fast energy-jump at the resonance. This novel energy-jump method was used to cross the coupling resonance and the results are summarized in Fig. 2 . In the experiment, an acceleration rate which was only $5 \%$ of the regular one was used to induce more spin flip at the intrinsic resonance $G_{\gamma}=0+\nu_{y}=8.8$, while an energy-jump at about half of the regular acceleration rate was generated to cross the coupling resonance at $G \gamma=0+\nu_{x}=8.6$. The energy-jump was accomplished by rapidly changing the beam circumference by $88 \mathrm{~mm}$ using the powerful AGS If system. Because of the large momentum spread of the beam indicated as a hashed band in the lower part of Fig. 2 , not all the beam particles are crossing the resonance during the jump unless the jump timing is carefully adjusted. From the beam momentum distribution, the ratio of the final to the initial polarization as a function of jump timing $T_{j \text { ump }}$ can be predicted and is shown as the solid line in the top half of Fig. 2. It shows good agreement with the data 
It clearly demonstrates that the novel energy-jump method can successfully overcome couping resonances and weak intrinsic resonances.

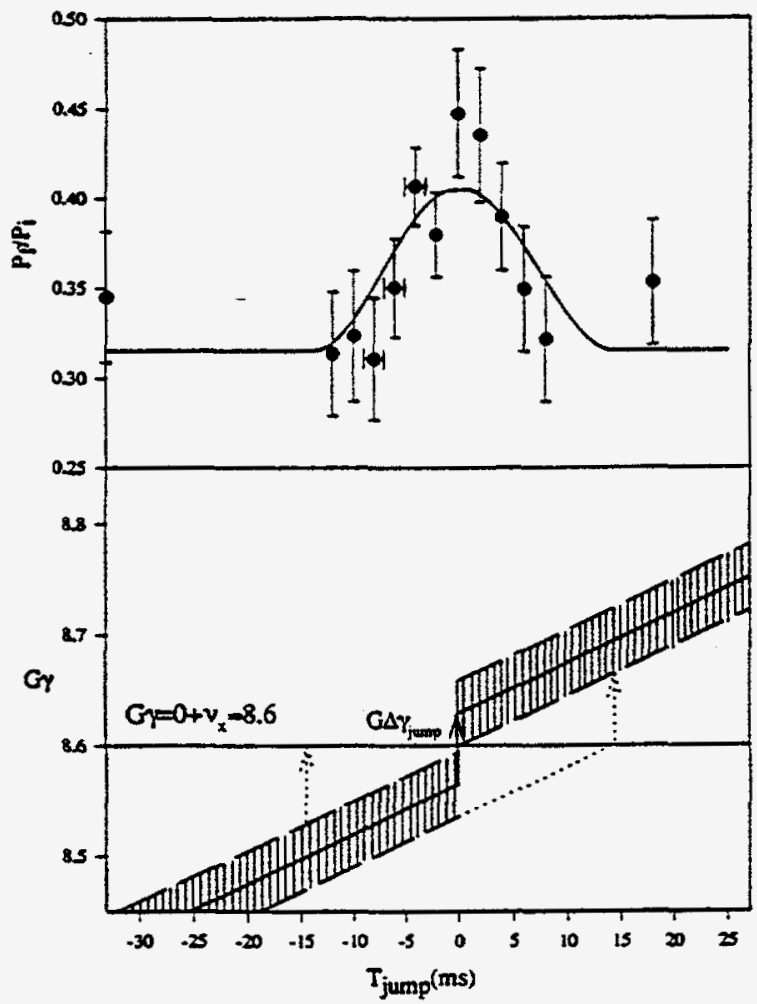

Figure 2: Energy jump data and schematics. In the top half, solid points are the experiment data, the solid line is the predicted curve, where $P_{f}$ is the beam polarization measured after crossing both $G \gamma=0+\nu_{x}=8.6$ and $G \gamma=0 \div \nu_{y}=8.8$, and $P_{i}$ is the beam polarization before the crossing. Bottom half shows the energy jump scheme. $G \gamma_{j u m p}$ is the amount of change of $G \gamma$ during the jump. The intrinsic resonance line $G \gamma=0+\nu_{y}=8.8$ is not shown.

\section{OUTLOOK}

In the furure, a novel scheme of overcoming strong intrinsic resonances is going to be tested, which is to use an $A C$ dipole[7], [8]. Full spin flip can be achieved with a stronger artificial AC spin resonance excited by an AC dipole at a modulation tune $\nu_{m}$. If we choose the AC spin resonance location $K_{A C}=n \pm \nu_{m}$ near the intrinsic spin resonance, the spin motion will be dominated by the AC dipole resonance and the spin near the intrinsic resonance will adiabatically follow the spin closed orbit of the AC spin resonance. With the AC dipole, a new dominant resonance near the intrinsic resonance is introduced, instead of enhancing the in trinsic resonance and aiso the coupling resonance strength, as has been proposed earlier[9]. Spin tracking studies for the AGS ring have indicated that this scheme should work. The energy-jump method can then be used for the residual coupling resonances and the weak intrinsic resonances.

\section{REFERENCES}

[1] L.H. Thomas, Phil. Mag. 3, I (1927); V. Bargmann, L. Michel, VI. Telegdi, Phys. Rev. Lett 2, 435 (1959).

[2] FZ. Khiari, et al., Phys. Rev. D39, 45 (1989).

[3] T. Roser, High Energy Spin Physics: 8th Intemational Symposium. Minneapolis in 1988, AIP Conference Proceedings, 187(1989) 1442.

[4] H. Huang, et al., Phys. Rev. Letr. 73, 2982 (1994).

[5] A. Luccio, BNL Report, BNL-52481(1995).

[6] H. Huang, T. Roser, A. Luccio, These Proceedings.

[7] M. Bai, et al.. These Proceedings.

[8] M. Bai, S.Y. Lee, H.Huang, T. Roser, M. Syphers, “Overcoming the Intrinsic Spin Resonance Using Resonance Island Created by RF Dipole", AGS/RHIC/SN No. 055.

[9] T. Roser, Proceedings of the 10th International Symposium on High Energy Spin Physics, Nagoya. (1992) 429. 


\section{DISCLAIMER}

This report was prepared as an account of work sponsored by an agency of the United States Government. Neither the United States Government nor any agency thereof, nor any of their employees, makes any warranty, express or implied, or assumes any legal liability or responsibility for the accuracy, completeness, or usefulness of any information, apparatus, product, or process disclosed, or represents that its use would not infringe privately owned rights. Reference herein to any specific commercial product, process, or service by trade name, trademark, manufacturer, or otherwise does not necessarily constitute or imply its endorsement, recommendation, or favoring by the United States Government or any agency thereof. The views and opinions of authors expressed herein do not necessarily state or reflect those of the United States Government or any agency thereof. 


\section{DISCLAMIER}

Portions of this document may be illegible in electronic inage products. Images are produced from the best available original document. 